\title{
Observations and analysis of two type IIP supernovae: the intrinsically faint object SN 2005cs and the ambiguous object SN 2005ay
}

\author{
D. Yu. Tsvetkov ${ }^{1}$, A. A. Volnova ${ }^{1}$, A. P. Shulga ${ }^{1}$, S. A. Korotkiy ${ }^{2}$,
} A. Elmhamdi ${ }^{3,4}$, I. J. Danziger ${ }^{4}$, and M. V. Ereshko ${ }^{2}$

\footnotetext{
${ }^{1}$ Sternberg State Astronomical Institute, Universitetskii pr. 13, 119992 Moscow, Russia e-mail: tsvetkov@sai.msu.su; elmhamdi@ts.astro.it

2 SC "Ka-Dar", Neopalimovskii 1st per. 16/13, 119121, Moscow, Russia

ICTP - International Centre for Theoretical Physics, Strada Costiera 11, 34014 Trieste, Italy

4 INAF - Osservatorio Astronomico di Trieste, via G.B.Tiepolo 11, 34131 Trieste, Italy

Received 26 May 2006 / Accepted 28 July 2006
}

\section{ABSTRACT}

\begin{abstract}
Aims. To derive observational properties and physical parameters of the progenitor stars of type IIP supernovae SN 2005ay and SN 2005cs from their $U, B, V, R, I$ CCD photometry, and to define their velocity behaviour.

Methods. Light curves are analysed, and the velocities and spectral characteristics of SN $2005 \mathrm{cs}$ are obtained using synthetic spectra modeling.

Results. Both supernovae are found to be fainter than the average SN IIP, with SN 2005cs being more subluminous and showing slight brightening in the second half of plateau stage in the $V, R, I$ bands and a low expansion velocity. The effects of two different plausible distance moduli on the derived physical parameters of SN 2005ay are considered. Two approaches are used to recover the amounts of the ejected ${ }^{56} \mathrm{Ni}$, indicating masses of the order of $\sim 0.02 M_{\odot}$, although late luminosities might indicate a higher amount for SN 2005ay, especially for the large distance case. Constraints on the progenitor properties are also presented, based on empirical analytical models. Two approaches are used to estimate the expansion velocities at the middle of the plateau phase. SN $2005 \mathrm{cs}$ represents an example of where all 3 physical parameters, velocity, energy and ${ }^{56} \mathrm{Ni}$ mass are lower than average, a correlation not always observed in SNe IIP. SN 2005ay may belong to the same class if the shorter distance possibility is adopted. Furthermore, the estimated mass range for SN 2005cs is in agreement with limits established by using pre-supernova imaging.
\end{abstract}

Key words. supernovae: general - supernovae: individual: SN 2005ay - supernovae: individual: SN2005cs

\section{Introduction}

In 2005 two bright type II supernovae (SN) were discovered in nearby spiral galaxies: SN 2005ay in NGC 3938 and SN 2005cs in M 51 (NGC 5194).

SN 2005ay was discovered on March 27 by D. Rich at magnitude 15.6 on CCD frames taken with a $0.31-\mathrm{m}$ reflector (IAUC 8500). The $\mathrm{SN}$ was located at $\alpha=11^{\mathrm{h}} 52^{\mathrm{m}} 48^{\mathrm{s}} .07, \delta=$ $+44^{\circ} 06^{\prime} 18^{\prime \prime} .4$ (equinox 2000.0), which is $15^{\prime \prime}$ west and $56^{\prime \prime}$ south of the center of Sc galaxy NGC 3938. Taubenberger et al. (2005) reported that a spectrum of SN 2005ay, obtained on March 29.98 UT with the Calar Alto 2.2-m telescope was that of a type-II SN soon (perhaps 8-10 days) after explosion. The spectrum was characterized by a blue continuum with broad P-Cyg features of the hydrogen Balmer series (the expansion velocity from $\mathrm{H} \beta$ was about $9600 \mathrm{~km} \mathrm{~s}^{-1}$ ).

The discovery of SN 2005cs was reported by W. Kloehr on June 28 at a magnitude of about 14 (IAUC 8553). W. Li confirmed the discovery and provided the position of the $\mathrm{SN}$ as $\alpha=13^{\mathrm{h}} 29^{\mathrm{m}} 53^{\mathrm{s}} 37, \delta=+47^{\circ} 10^{\prime} 28^{\prime \prime} .2$ (equinox 2000.0 ), which is 15" west and 67" south of the center of Sbc galaxy M51 (IAUC 8553). Nothing was visible at this location on earlier frames taken by W. Kloehr on May 11 and 26. A KAIT image taken on June 15.20 showed nothing at this position (limiting mag about 19.0), nor on Itagaki's image taken in poor conditions on June 20.598 (limiting mag 17.0).
Modjaz et al. (2005) reported that a spectrogram of SN 2005cs obtained on June 30.23 UT with the F. L. Whipple Observatory $1.5-\mathrm{m}$ telescope showed it to be a young type-II SN. The spectrum consisted of a blue continuum and P-Cyg profiles of the Balmer and He lines. The expansion velocity derived from the minimum of the $\mathrm{H} \beta$ line was about $7500 \mathrm{~km} \mathrm{~s}^{-1}$. Zerovelocity interstellar Na I D absorption with an equivalent width of about $0.02 \mathrm{~nm}$ was detected in the spectrum of SN 2005cs, indicating some reddening by dust. Interstellar $\mathrm{Na} I \mathrm{D}$ absorption was detected also at the redshift of the host galaxy, with comparable equivalent width, suggesting some host-galaxy extinction.

Maund et al. (2005) and Li et al. (2006) reported detection of the progenitor of SN $2005 \mathrm{cs}$ in pre-explosion archival images obtained with the Hubble Space Telescope. They concluded that it was a red supergiant of spectral type K0-M3, with initial mass $M_{\text {ZAMS }}=9_{-2}^{+3} M_{\odot}$. Li et al. also provided a provisional light curve for $\mathrm{SN}^{-2} 2005 \mathrm{cs}$ and supposed that it was a subluminous type IIP event.

\section{Observations and reductions}

The photometric observations were carried on with the 70-cm reflector of the Sternberg Astronomical Institute in Moscow (M70) using Apogee AP-47p (a) and AP-7p (b) CCD cameras, and with the $60-\mathrm{cm}$ reflector of the Crimean Observatory of the Sternberg 
Table 1. Colour terms for reduction equations.

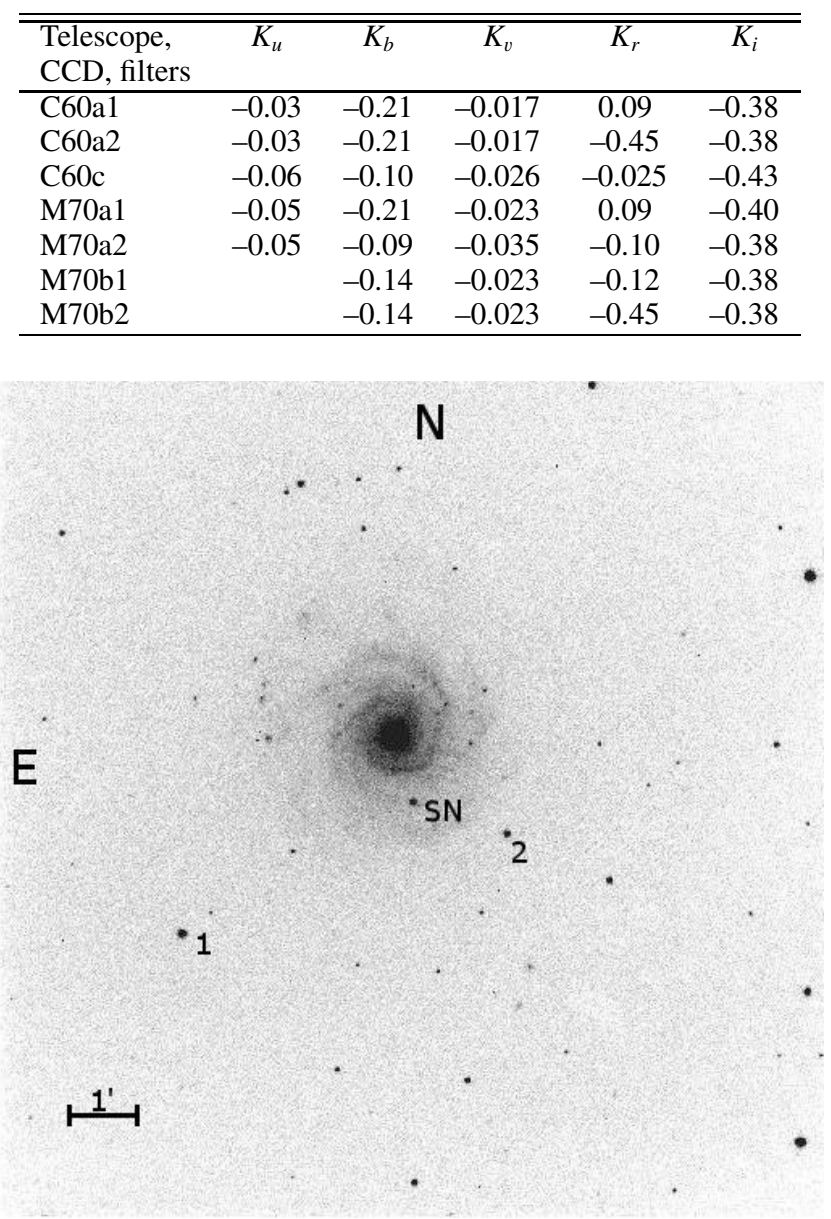

Fig. 1. SN 2005ay in NGC 3938 with comparison stars.

Astronomical Institute (C60) equipped with a Roper Scientific VersArray1300B (c) and Apogee AP-47p cameras. On July 14 observations were obtained at the 1-m telescope of the Special Astrophysical Observatory (S100) with CCD EEV42-40, and on July 11 at the $50-\mathrm{cm}$ meniscus telescope in the Crimea (C50) with a CCD Pictor416.

All reductions and photometry were made using $\mathrm{IRAF}^{1}$.

The colour terms for transformation of instrumental magnitudes $u b v r i$ to standard $U B V R_{\mathrm{c}} I_{\mathrm{c}}$ were determined for different telescope-filter-detector combinations using observations of standards in M 67 (Chevalier \& Ilovaisky 1991) and in NGC 7790 (Stetson 2000). The equations $u=U+K_{u}(U-B)+$ $C_{u} ; b=B+K_{b}(B-V)+C_{b} ; v=V+K_{v}(B-V)+C_{v} ; r=$ $R+K_{r}(V-R)+C_{r} ; i=I+K_{i}(R-I)+C_{i}$ were solved for colour terms, which are listed in Table 1 . The codes for telescopes and CCD cameras are followed by numbers denoting the filter sets. The colour terms for S100 were kindly given to us by V.P. Goranskij. Observations with the C50 were obtained only in the $V$ filter which was close to the standard system, and no correction was applied.

The images of the $\mathrm{SNe}$ with comparison stars are shown in Figs. 1, 2. The magnitudes of comparison stars were determined on photometric nights, when we observed standards from

${ }^{1}$ IRAF is distributed by the National Optical Astronomy Observatory, which is operated by AURA under cooperative agreement with the National Science Foundation.

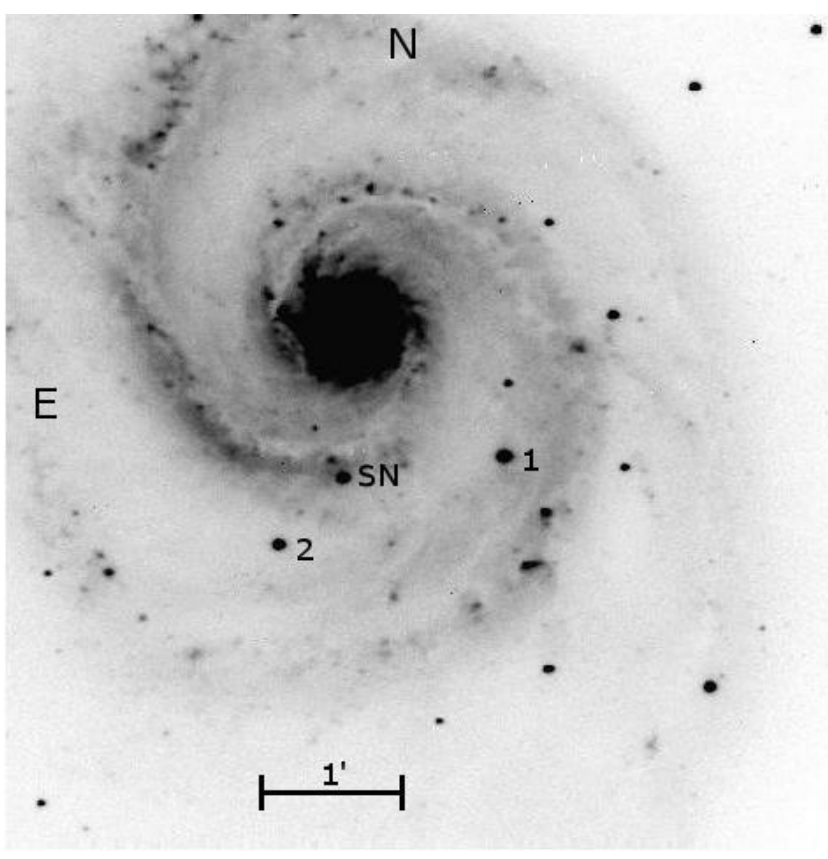

Fig. 2. SN 2005cs in M 51 with comparison stars.

Landolt (1992) and standard regions in the clusters M 67 and M 922. The magnitudes of stars M 51-1 and M 51-2 were derived by Richmond et al. (1996). We included their data in the calculation of mean values, which are presented in Table 2 with their RMS errors.

Photometric measurements of the $\mathrm{SNe}$ were made relative to comparison stars using PSF-fitting with the IRAF DAOPHOT package, and in some cases using aperture photometry with the APPHOT package. The background of the host galaxies around the SNe did not present any problems when the SNe were bright, but at late stages it could introduce additional errors. As the $\mathrm{SNe}$ are still visible on our images, we cannot apply image subtraction. Thus, the magnitudes at late stages can be regarded as provisional, and they should be verified later when images without the $\mathrm{SNe}$ are obtained and used for image subtraction. The results of SNe observations are presented in Tables 3-5.

Spectra of SN 2005cs were obtained on July 6 at the S100 with the UAGS spectrograph in the range 3425-6200 $\AA$ with a dispersion of $1.34 \AA$ per pixel. The spectra were extracted and wavelength and flux calibrated using the IRAF KPNOSLIT algorithm. The wavelength calibration was done using exposures of Ne lamp made before and after target observations. For flux calibration $\mathrm{BD}+28^{\circ} 4211$ was observed as a flux standard.

\section{The analysis}

SN 2005ay. We began to monitor this SN the day after discovery, on 2005 March 28, and the last image was obtained on 2006 April 26, more than a year after explosion. The light curves are shown in Fig. 3, where we also plotted the magnitude estimate by Kloehr on discovery images and the upper limit reported by Yamaoka \& Itagaki (2005). The light curves appear typical for type IIP SN, this being evident from comparison with the light curves of SN 1999gi (Leonard et al. 2002b). We shifted the light curves of SN 1999gi along the time axis to reach the best agreement for the stage of rapid decline after the plateau, and it is

\footnotetext{
${ }^{2}$ http://cadcwww.hia.nrc.ca/cadcbin/wdb/ astrocat/stetson/query/ NGC6341
} 
Table 2. Magnitudes of comparison stars.

\begin{tabular}{lcccccccccc}
\hline \hline Star & $U$ & $\sigma_{U}$ & $B$ & $\sigma_{B}$ & $V$ & $\sigma_{V}$ & $R$ & $\sigma_{R}$ & $I$ & $\sigma_{I}$ \\
\hline NGC 3938-1 & 14.47 & 0.05 & 14.40 & 0.03 & 13.83 & 0.02 & 13.49 & 0.02 & 13.17 & 0.02 \\
NGC 3938-2 & 16.36 & 0.08 & 15.86 & 0.03 & 15.07 & 0.02 & 14.63 & 0.02 & 14.25 & 0.02 \\
M51-1 & 14.19 & 0.04 & 14.03 & 0.01 & 13.41 & 0.01 & 13.07 & 0.01 & 12.76 & 0.02 \\
M51-2 & & & 16.42 & 0.05 & 15.11 & 0.02 & 14.35 & 0.01 & 13.71 & 0.02 \\
\hline
\end{tabular}

Table 3. BVRI photometry of SN 2005ay.

\begin{tabular}{cccccccccl}
\hline \hline JD 2 453 000+ & $B$ & $\sigma_{B}$ & $V$ & $\sigma_{V}$ & $R$ & $\sigma_{R}$ & $I$ & $\sigma_{I}$ & Tel. \\
\hline 458.45 & 15.32 & 0.09 & 15.12 & 0.08 & 14.98 & 0.05 & 15.02 & 0.09 & M70a1 \\
463.36 & 15.46 & 0.08 & 15.19 & 0.05 & 14.95 & 0.05 & 14.95 & 0.04 & M70a1 \\
465.42 & 15.47 & 0.06 & 15.20 & 0.06 & 14.94 & 0.07 & 14.94 & 0.05 & M70a1 \\
468.40 & 15.60 & 0.10 & 15.21 & 0.05 & 14.97 & 0.08 & 14.81 & 0.07 & M70a1 \\
473.40 & 15.76 & 0.06 & 15.16 & 0.04 & 14.94 & 0.04 & 14.80 & 0.05 & M70a1 \\
494.38 & 16.50 & 0.09 & 15.54 & 0.06 & 15.06 & 0.06 & 14.85 & 0.16 & M70b2 \\
495.32 & 16.37 & 0.07 & 15.33 & 0.06 & 15.04 & 0.04 & 14.70 & 0.12 & C60c \\
500.36 & 16.60 & 0.06 & 15.40 & 0.04 & 15.15 & 0.04 & 14.75 & 0.06 & C60c \\
506.41 & 16.53 & 0.08 & 15.45 & 0.06 & 15.02 & 0.06 & 14.74 & 0.11 & M70b2 \\
509.38 & 16.67 & 0.09 & 15.43 & 0.10 & 14.99 & 0.06 & 14.73 & 0.09 & M70b1 \\
515.37 & 16.67 & 0.08 & 15.42 & 0.10 & 15.03 & 0.07 & 14.73 & 0.11 & M70b1 \\
560.34 & 17.65 & 0.11 & 16.33 & 0.04 & 15.75 & 0.04 & & & C60a2 \\
562.31 & 18.10 & 0.14 & 16.56 & 0.04 & 15.83 & 0.03 & 15.44 & 0.10 & C60a2 \\
566.30 & & & 16.98 & 0.08 & 16.30 & 0.03 & 15.80 & 0.11 & C60a2 \\
566.31 & 18.66 & 0.05 & 17.14 & 0.04 & 16.42 & 0.03 & 15.88 & 0.04 & S100 \\
569.29 & 18.61 & 0.14 & 17.37 & 0.10 & 16.58 & 0.05 & 16.33 & 0.15 & C60a2 \\
680.63 & & & 18.67 & 0.08 & 17.71 & 0.05 & & & C60a1 \\
683.58 & 19.90 & 0.10 & 18.76 & 0.06 & 17.81 & 0.04 & 17.62 & 0.11 & C60a1 \\
687.57 & 19.83 & 0.15 & 18.70 & 0.06 & 17.81 & 0.09 & & & C60a1 \\
688.55 & & & 18.85 & 0.16 & 17.74 & 0.12 & & & C60a1 \\
804.56 & & & & & 18.83 & 0.28 & & & M70a1 \\
852.37 & & & & & 19.36 & 0.24 & & & M70a2 \\
\hline
\end{tabular}

Table 4. Observations of SN 2005cs.

\begin{tabular}{cccccccccl}
\hline \hline JD 2 453000+ & $B$ & $\sigma_{B}$ & $V$ & $\sigma_{V}$ & $R$ & $\sigma_{R}$ & $I$ & $\sigma_{I}$ & Tel. \\
\hline 555.33 & 14.45 & 0.02 & 14.45 & 0.02 & 14.30 & 0.02 & 14.37 & 0.05 & C60a2 \\
558.32 & 14.57 & 0.02 & 14.54 & 0.02 & 14.34 & 0.02 & 14.29 & 0.03 & C60a2 \\
561.32 & 14.74 & 0.02 & 14.50 & 0.02 & 14.26 & 0.02 & 14.25 & 0.03 & C60a2 \\
563.30 & & & 14.50 & 0.02 & & & & & C50 \\
564.29 & 14.92 & 0.02 & 14.50 & 0.02 & 14.32 & 0.02 & 14.20 & 0.03 & C60a2 \\
565.36 & 15.08 & 0.05 & 14.65 & 0.04 & 14.42 & 0.04 & 14.29 & 0.06 & M70b1 \\
566.33 & 15.19 & 0.02 & 14.63 & 0.02 & 14.39 & 0.02 & 14.32 & 0.03 & S100 \\
567.29 & 15.13 & 0.03 & 14.60 & 0.02 & 14.34 & 0.02 & 14.23 & 0.03 & C60a2 \\
571.29 & 15.40 & 0.02 & 14.72 & 0.02 & 14.43 & 0.02 & 14.27 & 0.03 & C60a2 \\
571.40 & 15.34 & 0.02 & 14.72 & 0.02 & 14.38 & 0.04 & 14.22 & 0.04 & M70b1 \\
580.32 & 15.69 & 0.05 & 14.73 & 0.03 & 14.42 & 0.03 & 14.20 & 0.04 & M70b1 \\
587.31 & 15.77 & 0.05 & 14.72 & 0.03 & 14.31 & 0.02 & 14.08 & 0.03 & M70b1 \\
593.31 & 15.82 & 0.03 & 14.72 & 0.03 & 14.28 & 0.02 & 14.03 & 0.03 & M70b1 \\
607.34 & 15.89 & 0.06 & 14.63 & 0.12 & 14.15 & 0.07 & 13.89 & 0.08 & M70b1 \\
612.29 & 16.08 & 0.03 & 14.74 & 0.02 & 14.14 & 0.02 & 13.87 & 0.05 & C60a1 \\
613.36 & & & 14.63 & 0.06 & 14.14 & 0.05 & 13.84 & 0.13 & C60a1 \\
618.28 & 15.99 & 0.06 & 14.71 & 0.05 & 14.16 & 0.03 & & & C60c \\
620.25 & & & 14.68 & 0.03 & 14.12 & 0.02 & 13.83 & 0.04 & C60c \\
621.25 & 16.15 & 0.08 & 14.84 & 0.07 & 14.15 & 0.03 & 13.80 & 0.06 & M70b1 \\
628.25 & 16.11 & 0.04 & 14.79 & 0.04 & 14.17 & 0.04 & 13.83 & 0.05 & M70b1 \\
644.19 & 16.53 & 0.19 & 14.96 & 0.11 & 14.29 & 0.05 & 13.96 & 0.10 & M70b1 \\
653.24 & 16.37 & 0.13 & 15.04 & 0.15 & 14.39 & 0.08 & 14.03 & 0.12 & M70b1 \\
683.63 & & & 17.91 & 0.15 & 17.20 & 0.11 & 16.58 & 0.09 & C60a1 \\
687.60 & & & 17.77 & 0.19 & 17.22 & 0.14 & & & C60a1 \\
804.56 & & & 18.62 & 0.18 & 17.81 & 0.11 & & & M70a2 \\
831.43 & & & 18.41 & 0.11 & 18.07 & 0.10 & & & M70a2 \\
850.46 & & & 18.59 & 0.15 & 18.30 & 0.09 & 17.62 & 0.14 & M70a1 \\
\hline
\end{tabular}


Table 5. Observations of SNe in the $U$ band.

\begin{tabular}{ccclcccl}
\hline \hline \multicolumn{3}{c}{ SN 2005ay } & \multicolumn{5}{c}{ SN 2005cs } \\
\hline JD 2 453000+ & $U$ & $\sigma_{U}$ & Tel. & JD 2453000+ & $U$ & $\sigma_{U}$ & Tel. \\
\hline 458.450 & 14.67 & 0.11 & M70a1 & 566.33 & 15.36 & 0.06 & S100 \\
463.360 & 14.84 & 0.21 & M70a1 & & & & \\
465.420 & 15.01 & 0.20 & M70a1 & & & & \\
495.320 & 17.58 & 0.16 & C60c & & & & \\
500.360 & 17.57 & 0.10 & C60c & & & & \\
\hline
\end{tabular}

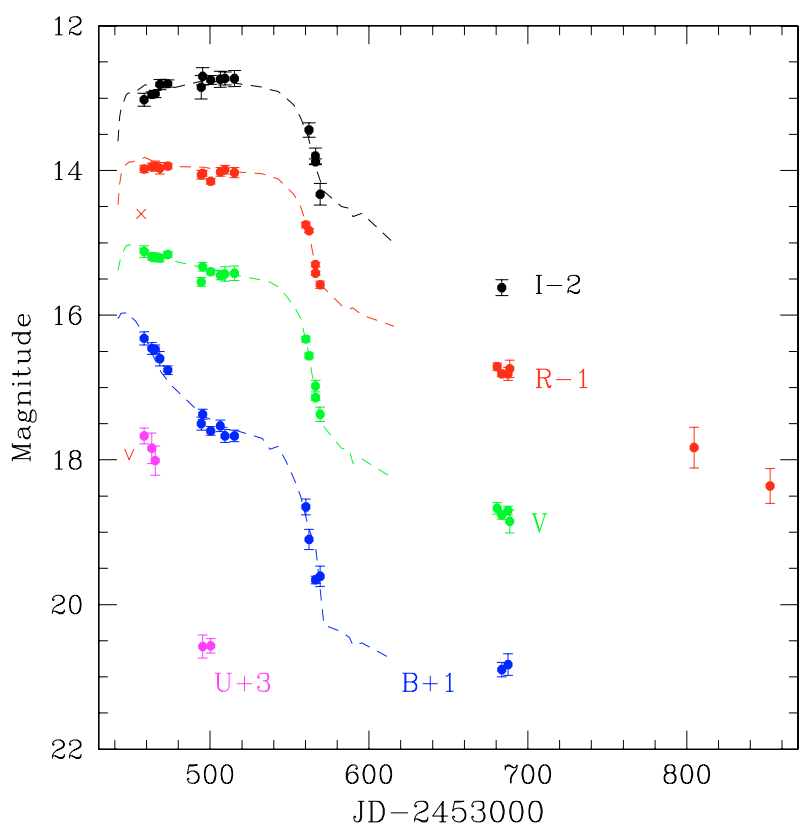

Fig. 3. $U B V R I$ light curves of SN 2005ay. Dots show our data, cross is for the discovery magnitude by Rich (2005), the " $v$ " mark is the upper limit from Yamaoka \& Itagaki (2005). The dashed lines are the light curves of SN 1999gi.

clear that the plateau phase was shorter for SN 2005ay ( $10 \%$ lower plateau duration). At this stage the brightness in the $R$ band was constant, but we can notice slight brightening in $I$ towards the middle of the plateau, and a decline of luminosity in $V$ at a rate of about $0.006 \mathrm{mag} / \mathrm{day}$. In the $B$ and $U$ bands the luminosity decreased steadily, and the rate of decline in $U$ amounted to $0.08 \mathrm{mag} / \mathrm{day}$. After the plateau phase, the light curves show a rapid drop of about 2 mag, followed by a linear decline in magnitude. We can estimate the rate of decline only in the $R$ band, and it is very close to the standard value of $0.01 \mathrm{mag} / \mathrm{day}$, similar to the radioactive decay of ${ }^{56} \mathrm{Co}$.

We estimate the following magnitudes at maximum light, which was reached at the onset of the plateau in all bands except $I: U_{\max }=14.7, B_{\max }=15.3, V_{\max }=15.2, R_{\max }=$ $15.0, I_{\max }=14.7$. The light of SN 2005ay suffered minor extinction in the Galaxy: $A_{B}=0.09$ according to Schlegel et al. (1998). We estimate the total extinction by comparing the colour curves of SN 2005ay with those for SN 1999gi. The comparison shows that in the first 20 days after discovery the $(B-V)$ colour of SN 2005ay is very close to SN 1999gi, being only 0.04 mag bluer. But at the phase 35-60 days SN 2005ay is significantly bluer than SN 1999gi, by about 0.13 mag. Thus there is an intrinsic difference in the observed colour curves of these $\mathrm{SNe}$, and we suggest that the colour excess for SN 2005ay was about $0.1 \mathrm{mag}$ less than for SN 1999gi. As the total colour excess was estimated as $E(B-V)=0.21 \pm 0.09 \mathrm{mag}$ for SN 1999gi

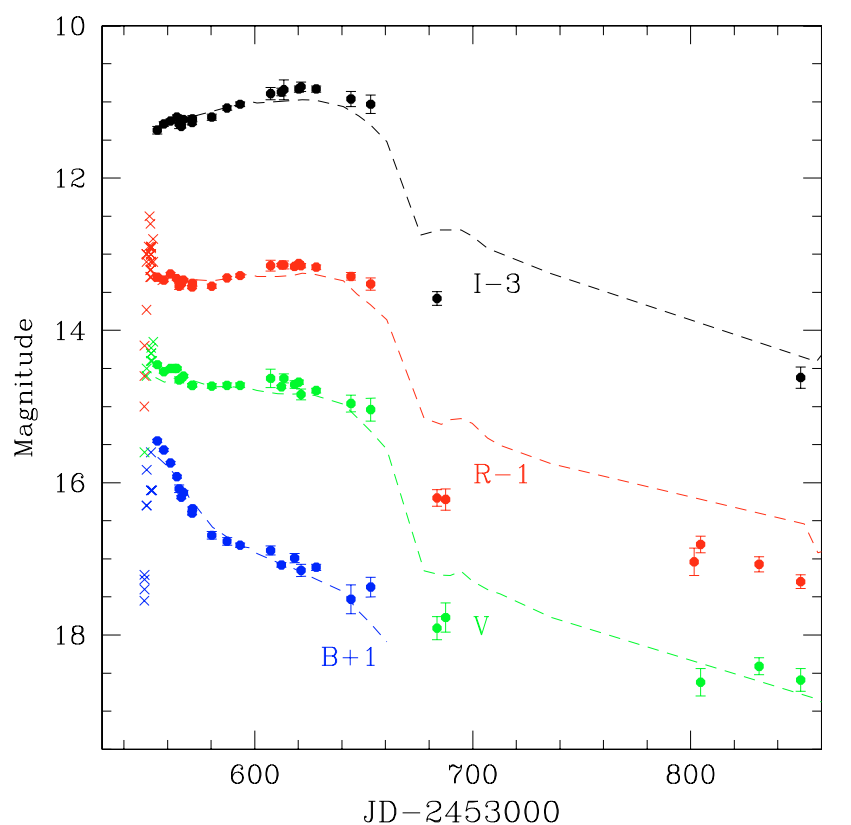

Fig. 4. BVRI light curves of SN 2005cs. Dots show our data, crosses are for the observations of amateur astronomers. The dashed lines are the light curves of SN 1999em.

(Leonard et al. 2002), we propose $E(B-V) \approx 0.1 \mathrm{mag}$ for SN 2005ay. Using this estimate the corrected-intrinsic $(B-V)$ colour curves are illustrated in Fig. 6 (bottom panel). Adopting a distance modulus $\mu=30.82$ from the "LEDA" database ${ }^{3}$, we derive absolute magnitudes at a maximum of $M_{B} \simeq M_{V} \simeq-15.9$. This is about 0.8 mag fainter than the mean absolute magnitude for SNe IIP from Richardson et al. (2002) recalculated to the value of $H_{0}=70 \mathrm{~km} \mathrm{~s}^{-1} \mathrm{Mpc}^{-1}$. We note here that NGC 3938 belongs to the Ursa Major group. The spiral galaxy NGC 3982, which is also part of the group, has a Cepheid calibrated distance modulus of 31.71 (Saha et al. 2001). If assuming the two galaxies have this same distance, then SN 2005ay would be intrinsically more luminous, which would increase the synthesized ${ }^{56} \mathrm{Ni}$ mass by a factor of $\sim 2.26$ (see Sect. 4 ). The front-to-back effects on the relative distances in the group remain uncertain. The significance of the difference in recession velocity of the two galaxies $(\sim 0.0037$ and $\sim 0.0027$ for NGC 3982 and NGC 3938, respectively; LEDA database) also remains uncertain.

SN 2005cs. We started observations of this object on July 3 , 5 days after discovery, and continued monitoring until 2006 April 24. The light curves are shown in Fig. 4, where we also plotted data from amateur astronomers reported on the Astrosurf site $^{4}$, obtained before our first data point. For comparison we

\footnotetext{
${ }^{3}$ http://leda.univ-lyon $1 . \mathrm{fr} /$

${ }^{4}$ http://www . astrosurf. com/snweb2/2005/ 05cs/05csMeas.htm
} 


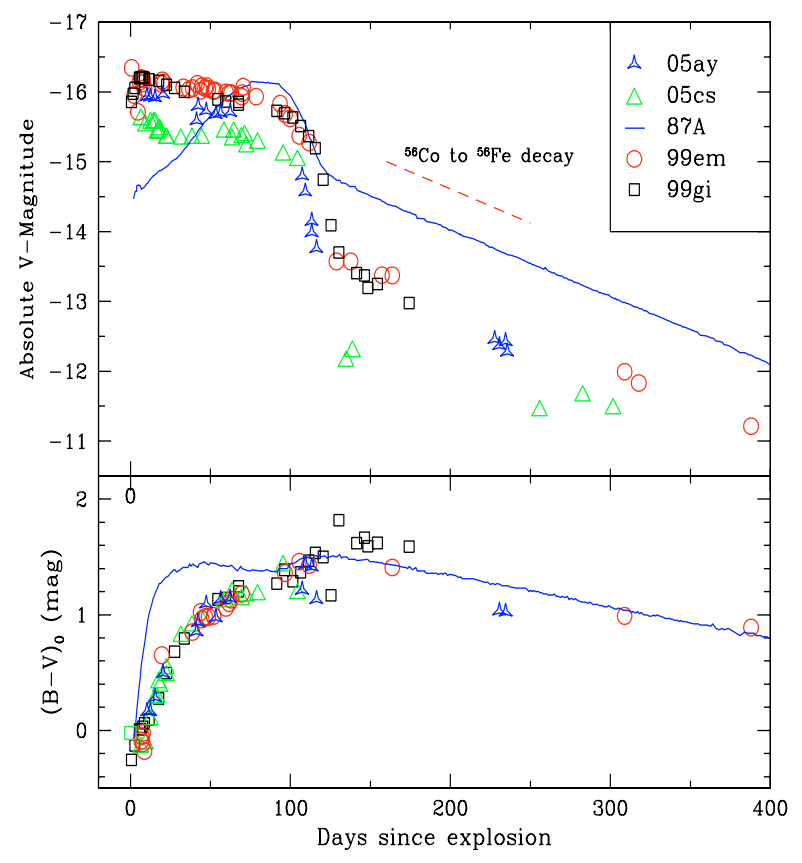

Fig. 5. Upper panel: the absolute $V$-light curves of SNe 2005ay (shorter distance case) and 2005cs, compared to SNe 1987A ( $D=50 \mathrm{kpc}, A_{\mathrm{V}}^{\text {tot }}=$ $0.6 \mathrm{mag}), 1999 \mathrm{em}\left(D=8.8 \mathrm{Mpc} ; A_{\mathrm{v}}^{\text {tot }}=0.31 \mathrm{mag}\right)$ and $1999 \mathrm{gi}$ $\left(D=10.91 \mathrm{Mpc} ; A_{V}^{\text {tot }}=0.65 \mathrm{mag}\right)$. For SN 2005ay we plot the shorter distance case. Lower panel: the " $(B-V)$ " intrinsic colour evolution of the $\mathrm{SNe}$ sample.

plotted the light curves for a typical SN IIP 1999em (Leonard et al. 2002a; Elmhamdi et al. 2003a; Hamuy et al. 2001). SN 2005 cs is certainly of type IIP, but the light curves are noticeably different from those of SN 2005ay. The early magnitudes from amateurs, taken by different and independent obsevers, suggest the presence of a narrow peak before the onset of the plateau in the $V$ and $R$ bands at JD $2453551-552$, but we cannot exclude that it is due to the errors of their data. Nevertheless, we can conclude that the fast brightening finished at that epoch, and that maximum light in the $B$ band was reached on JD 2453553 with $B_{\max }=14.5 \mathrm{mag}$. In the $V$ and $R$ bands the luminosity declined slowly until JD 2453580 , and then a slight brightening is seen during the plateau phase. At the same epoch the rate of decline in $B$ slowed down. In the $I$ band, the brightness of the $\mathrm{SN}$ increased from the onset of the plateau stage and maximum light in $I$ and $R$ was reached on JD 2453620 , with $R_{\max }=14.15, I_{\max }=13.80$. In the $V$ band, SN $2005 \mathrm{cs}$ was brightest at a very early epoch, with $V_{\max }=14.3$. A similar behavior was observed for SN 1999em at the plateau stage, but the increase in brightness was more pronounced for SN $2005 \mathrm{cs}$. The plateau stage lasted until about day JD 2453660 , and then the light curves display a steep decline. The luminosity dropped by about $2.8 \mathrm{mag}$, the decline was significantly greater than for SN 1999em, as suggested by Li et al. (2006). The final exponential tail started at about JD 2453680 , and the decline rate in $R$ was $\sim 0.007 \mathrm{mag} /$ day. In the other bands the quality of our data does not allow to estimate the decline rate (see Sect. 4 for discussions about late $V$-photometry and ${ }^{56} \mathrm{Ni}$ estimates).

According to Modjaz et al. (2005) and Li et al. (2006), the most likely value of total colour excess for $\mathrm{SN} 2005 \mathrm{cs}$ is $E(B-V) \approx 0.1$. A more detailed view regarding the colour excess for SN 2005cs has been presented by Maund et al. (2005) The analysis suggests an amount of total reddening of $E(B-V)=$ $0.14 \pm 0.2$, which we adopt in our present work. The $(B-V)$

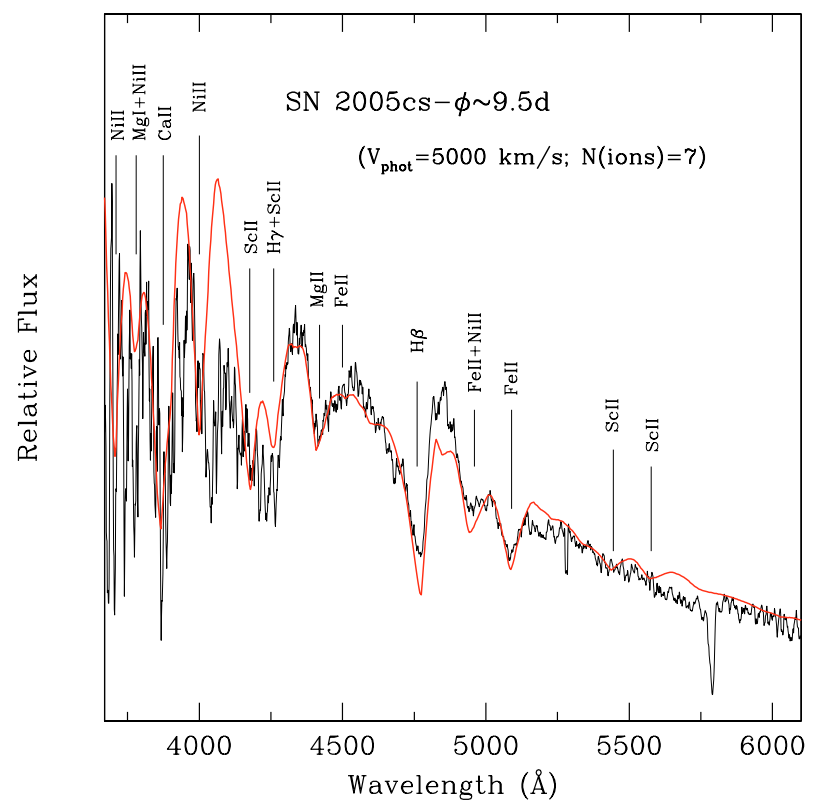

Fig. 6. Spectrum of $\mathrm{SN} 2005 \mathrm{cs}$ obtained on July 6, 2005 (JD 2453558.37 ) at S100, compared with the SYNOW synthetic spectrum (thin line). Lines that are responsible for the most conspicuous features are indicated.

colour evolution is shown in Fig. 5 (lower panel), and compared to SNe 1987A, 1999em, 1999gi and 2005ay. Taking this value for extinction and a distance modulus of $\mu=29.6$ (Feldmeier et al. 1997; Maund et al. 2005), SN 2005cs is an underluminous type IIP SN, more than a magnitude fainter than the mean IIP luminosity (Richardson et al. 2002) (see details in Sect. 4).

The spectrum of SN 2005cs obtained on July 6 (JD 2453 558.37) is presented in Fig. 6. The $\mathrm{H} \beta$ and $\mathrm{H} \gamma$ features are prominent with typical P-Cygni profiles, and Fe II absorption lines are also evident, with observed absorption minima at $4955 \AA$ and $5094 \AA$. From the wavelengths of $\mathrm{H} \beta$ and Fe II $\lambda 5169$ absorption minima, we can derive expansion velocities of 5575 and $4984 \mathrm{~km} \mathrm{~s}^{-1}$, taking $600 \mathrm{~km} \mathrm{~s}^{-1}$ as the recession velocity of $M 51$. A spectrum analysis was made by means of the synthetic code SYNOW in Sect. 4.2. We see that the expansion has slowed down by about $2000 \mathrm{~km} \mathrm{~s}^{-1}$ since the observation by Modjaz et al. (2005). These velocities are significantly lower than the values for SN 1999em (Leonard et al. 2002a) at similar epochs.

\section{Main physical properties}

\subsection{Estimate of ejected ${ }^{56} \mathrm{Ni}$ mass}

The absolute $V$-band light curves of SNe 2005ay and 2005cs are illustrated in Fig. 5 (upper panel). For SN 2005ay, the shorter distance is used in the plot. The light curves of the peculiar SN 1987A and SNe IIP 1999em and 1999gi are displayed for comparison. For the ${ }^{56} \mathrm{Ni}$ mass determination the total extinction and distance estimates are crucial. The adopted parameters for the well studied SNe 1987A, 1999em and 1999gi are listed on the caption of Fig. 5. The assumed explosion time also affects the ${ }^{56} \mathrm{Ni}$ mass estimates. For SN $2005 \mathrm{cs}$ the explosion time is estimated to be June 27.3 (JD 2453548.8 ), or earlier by as much as 1.5 days. This is based on the early rise in the light curves, especially in the $B$-band, from our data combined with the online 
Table 6. Main parameters of the SNe.

\begin{tabular}{cccccc}
\hline \hline SN & Host & Explosion & Distance & $E(B-V)$ & $\mathrm{M}_{V}{ }^{1}$ \\
name & Galaxy & time (JD) & modulus & (total) & at $\sim 300 \mathrm{~d}$ \\
\hline 2005ay & NGC 3938 & 2453453 & $30.82-31.71$ & 0.1 & $-11.78--12.67$ \\
2005cs & M 51 & 245348.8 & 29.62 & 0.14 & -11.46 \\
\hline
\end{tabular}

1- Extrapolated at day 300 for comparison.

Estimates adopting short and large distances are reported for SN 2005ay.

Astronomers data ${ }^{5}$. Following the discussion by Maund et al. (2005), we adopt a total colour excess and distance to the host galaxy of $E(B-V)=0.14 \pm 0.2$ and $8.4 \mathrm{Mpc}$, respectively.

The situation of SN 2005ay is less clear. On the one hand, the very early photometry of the rise is lacking, although our earliest data indicate a slight trend of increase in the apparent luminosity in the $R$ and $I$ bands. On the other hand, the similarity to the SN 1999gi light curves is evident, both on the plateau and on the transition phase to reach the radioactive tail (Fig. 3). The SN 1999gi light curve shape could be used to fit SN 2005ay data and hence obtain an estimate of the explosion epoch. This leads to $t_{0} \simeq$ March 13 (JD=2 453 443). However a CCD image taken on March 19.621 (JD=2 453449.62$)$ showed nothing at the location of the supernova (Yamaoka \& Itagaki 2005). This analysis therefore demonstrates the incorrectness of estimating explosion times based on light curve fits, even for seemingly similar events within homogeneous class of supernovae such as type IIP. A direct consequence of this reasoning is that SN 2005ay has a short plateau compared to SN 1999gi, implying possible differences in the physical parameters, especially the ejecta mass, athough the velocity behaviour and the bolometric plateau luminosity also must be considered.

The early evolution of the intrinsic $(B-V)$ colour can provide further constraints on the explosion time. Assuming a total colour excess of $E(B-V)=0.1$ towards SN 2005ay, we estimate the explosion time to be $t_{0} \simeq$ March $23(\mathrm{JD}=2453453)$, with an uncertainty of 2 days. This is based on the assumption of a similar photospheric-phase temperature evolution behaviour in type IIP objects, which is related to the adiabatic cooling of the radiation trapped in the expanding envelope (Schmidt et al. 1992; Elmhamdi et al. 2003b). The difference to SN 1987A is indeed due to a faster cooling due to the smaller presupernova radius (i.e. the blue supergiant nature of the progenitor star). The standard reddening laws of Cardelli et al. (1989) are adopted. Figure 5 (bottom panel) displayed the intrinsic colour curves of the five SNe. During the first 100 days the similarity between the $\mathrm{SNe}$, except SN 1987A, gives us confidence in the assumed parameters and hence the reliability of the absolute $V$-light curves that will be used later for the estimation of ${ }^{56} \mathrm{Ni}$ mass. The main parameters of the two events are summarized in Table 6 .

The absolute $V$-light curves of SNe 2005ay and 2005cs, Fig. 5 (top panel), highlight the different phases of evolution compared to SNe 1987A, 1999em and 1999gi, during the first 300 days. At early times SN 2005cs shows a brief peak followed by a settling onto the plateau phase, around day 18 after explosion, in which the magnitude remains almost constant, accounting for the cooling and the expansion balance. An absolute magnitude of $M_{v} \simeq-15.33$ is measured from a least squares fit of the plateau data (from $+18 \mathrm{~d}$ to $+105 \mathrm{~d}$ ). Later the light curve exhibits a steep decline, $\sim 2.8$ mag in $\sim 30$ days, signaling the onset of the nebular phase and the start of the exponential tail. The

\footnotetext{
5 http://www . astrosurf. com/snweb2/2005/05cs/ Q5csMeas.htm
}

radioactive decay phase is clearly not well sampled, nevertheless one may remark a slower decay compared to the ${ }^{56}$ Co slope (dashed line in Fig. 5). Similar flattening has been reported in intrinsically fainter objects (Elmhamdi et al. 2003a). In the cited work, it has also been noted that the duration of this flattening period, seen also in other bands, might correlate with the synthesized ${ }^{56} \mathrm{Ni}$ mass, with lower ${ }^{56} \mathrm{Ni}$ mass objects having a longer duration flattening. The last two photometric points show a decline rate close to the ${ }^{56} \mathrm{Co}$ decay. Therefore we rely on these late data to recover the amount of the ejected ${ }^{56} \mathrm{Ni}$. Adopting a ${ }^{56} \mathrm{Ni}$ mass of $0.075 M_{\odot}$ for SN 1987A (Catchpole et al. 1988; Bouchet \& Danziger 1993), a direct shift of the light curve tail of SN 1987A to fit late data of SN 2005cs indicates a synthesized amount of ${ }^{56} \mathrm{Ni}$ of $\sim 0.017 M_{\odot}$.

The $M_{V}$ photometry observations of SN 2005ay indicate an early decline from a short maximum (lasting about 17 days with $M_{V}^{\max } \simeq-15.94$ for the shorter distance and -16.83 for the larger one), reaching the plateau phase with a measured mean absolute magnitude of about $M_{\mathrm{V}} \simeq-15.72$ and -16.61 for the shorter and longer distances, respectively. Our photometry is poorly sampled at the end of the plateau phase, however the data at the transition phase plateau-tail seem to indicate a shorter plateau phase compared to SNe 2005cs and 1999em. The observed data, around day 230 after explosion, span about 8 days of observations. These late data are used as above to recover the ${ }^{56} \mathrm{Ni}$ mass (i.e. best fit with SN 1987A radioactive tail). This method leads to $\sim 0.023 M_{\odot}$ for the shorter distance and $\sim 0.051 M_{\odot}$ for the larger distance.

As a further check of the derived ${ }^{56} \mathrm{Ni}$ masses of the two events, we make use of the well established correlation between the ${ }^{56} \mathrm{Ni}$ mass and the absolute $M_{V}$ at the plateau phase (Elmhamdi et al. 2003b; Hamuy 2003). Adopting the previous estimates of $M_{V}$ during the plateau phase, i.e. $M_{V} \simeq-15.33$ for SN $2005 \mathrm{cs}$ and $M_{V} \simeq-15.72 ;-16.61$ for SN 2005ay, the method gives ${ }^{56} \mathrm{Ni}$ masses of $\sim 0.018 M_{\odot}$ for $\mathrm{SN} 2005 \mathrm{cs}$ and $\sim 0.026 M_{\odot}$ for the shorter distance and $\sim 0.065 M_{\odot}$ for the larger distance for SN 2005ay.

It is reassuring that the two methods give consistent results and indicate rather low ejected ${ }^{56} \mathrm{Ni}$ masses for SN $2005 \mathrm{cs}$ and for SN 2005ay if the shorter distance obtains. A higher amount for SN 2005ay obtains for the larger distance. This indicates that at least SN 2005cs belongs to the faint tail of the luminosity function of type II SNe while the situation for SN 2005ay depends on the adopted distance.

\subsection{The progenitor characteristics}

Making use of the type IIP-analytical model of Popov (1993), and the hydrodynamic models of Litvinova \& Nadyozhin (1985), it is possible to derive physical parameters, namely en$\operatorname{ergy}(E)$, ejecta mass $\left(M_{\mathrm{ej}}\right)$ and presupernova radius $(R)$. The duration of the plateau $\left(t_{\mathrm{p}}\right)$, the absolute $V$-magnitude at the plateau phase $\left(M_{\mathrm{v}}\right)$ and the photospheric velocity $\left(V_{\mathrm{p}}\right)$ are the input parameters, usually estimated from the observations. 
Table 7. The SNe progenitor properties.

\begin{tabular}{ccccccc}
\hline \hline & & SN 2005ay & \multicolumn{3}{c}{ SN 2005cs } \\
\hline $\begin{array}{c}t_{\text {plateau }} \\
\text { (days) }\end{array}$ & $\begin{array}{c}M \text { ej } \\
\left(M_{\odot}\right)\end{array}$ & $\begin{array}{c}E \\
\left(10^{51} \mathrm{erg} / \mathrm{s}\right)\end{array}$ & $\begin{array}{l}R \\
\left(R_{\odot}\right)\end{array}$ & $\begin{array}{c}M \text { ej } \\
\left(M_{\odot}\right)\end{array}$ & $\begin{array}{c}E \\
\left(10^{51} \mathrm{erg} / \mathrm{s}\right)\end{array}$ \\
$\left(R_{\odot}\right)$
\end{tabular}

Ejected ${ }^{56} \mathrm{Ni}$ mass:

$0.023-0.026 M_{\odot}($ shorter distance $)$

$0.051-0.065 M_{\odot}$ (larger distance)

$0.017-0.018 M_{\odot}$

SN 2005ay: $M_{V}($ Plateau $)=-15.72 \& V_{\text {phot }}=2980 \mathrm{~km} \mathrm{~s}^{-1}$ (shorter distance);

$M_{V}($ Plateau $)=-16.61 \& V_{\text {phot }}=4070 \mathrm{~km} \mathrm{~s}^{-1}$ (larger distance) .

SN 2005cs: $M_{V}($ Plateau $)=-15.33 \& V_{\text {phot }}=2600 \mathrm{~km} \mathrm{~s}^{-1}$.

As discussed previously, from observations of SNe 2005ay and $2005 \mathrm{cs}$ we adopt $M_{V}($ plateau $) \simeq-15.72 ;-16.61$ and $M_{V}($ plateau $) \simeq-15.33$, respectively. The early rise seen in both light curves of the two objects give constraints on the duration of the plateau (starting later than 2 weeks after explosion). We adopt a range of $t \mathrm{p}=80-90$ days for the two events, although SN 2005ay clearly has a shorter plateau duration than SN 2005cs. This range represents our estimate of the uncertainty associated with the determination of this parameter. We note that more precise constraints on the plateau duration should come from an integrated bolometric light curve shape. The obvious observed initial declines in $U$ and $B$ bands would affect the resulting constructed bolometric light curves, indicating a shorter plateau phase than is seen in the $V$-band light curve (for expample SN 1999em; Elmhamdi et al. 2003a)

The third input parameter is the representative photospheric velocity during the plateau phase. In principal this should be estimated from weak lines in the observed spectra during the plateau epoch. Unfortunately we lack such observations for the two SNe. However, an available spectrum of SN $2005 \mathrm{cs}$ is observed around 9.5 days since explosion. In order to analyse this spectrum we use the parametrized supernova synthetic spectra code SYNOW. Details of the fitting procedure and parameters can be found elsewhere (see for example Elmhamdi et al. 2006). Figure 6 showed the observed spectrum, after corrections for reddening and host galaxy recession velocity, compared to the best fit synthetic spectrum that has a blackbody temperature of $T_{\mathrm{bb}}=14500 \mathrm{~K}$, a photospheric velocity of $V_{\text {phot }}=5000 \mathrm{~km} \mathrm{~s}^{-1}$, and including 7 ions, namely: H I, Fe II, Ca II, Mg II, Mg I, Sc II and Ni II. Synthetic line profile features are labeled by the designation of the ion. The observed spectrum has been smoothed with a box size of 6 (in pixels). The modeling has been undertaken adopting an exponential radial dependence of the optical depth (and density), $\propto \exp \left(-v / v_{\mathrm{e}}\right)$, with an $e$-folding velocity $v_{\mathrm{e}}=2000 \mathrm{~km} \mathrm{~s}^{-1}$. The match is satisfactory as the main features are accounted for by the synthetic spectrum. On one hand, the good fit with Fe II, especially Fe II $5169 \AA$ A, gives us confidence in the deduced photospheric velocity. On the other hand, the Balmer $\mathrm{H}$ I features, especially $\mathrm{H} \beta$, are clearly identified, and found to have a velocity $V_{\min }(\mathrm{HI})=5500 \mathrm{~km} \mathrm{~s}^{-1}$. The supernova has a low velocity behaviour with clearly narrow features. Indeed, at similar phases the well observed SNe II $1987 \mathrm{~A}$ and $1999 \mathrm{em}$ had velocities about $4000 \mathrm{~km} \mathrm{~s}^{-1}$ higher. We note here that an expansion velocity of about $7500 \mathrm{~km} \mathrm{~s}^{-1}$ has been derived from the minimum of the $\mathrm{H} \beta$ from the published
SN 2005cs CFA-spectrum 3 days after explosion (Modjaz et al. 2005). Again, at a similar age, H I Balmer lines in SN 1987A had an expansion velocity of the order of $16000 \mathrm{~km} \mathrm{~s}^{-1}$. We note also that in their spectrum there occur features reminiscent of what was ascribed to N II lines in SN 1999em (Baron et al. 2000). Our SYNOW test modeling of the CFA-spectrum suggests that N II lines could be the correct interpretation also for SN $2005 \mathrm{cs}$.

For our purpose, i.e. analytical models, we need an appropriate estimate of the photospheric velocity during the photospheric phase. We propose two different methods, based on two established results in type IIP SNe. First, the correlation $M_{V}(50 \mathrm{~d})$ vs. $V_{\text {phot }}(50 \mathrm{~d})$ that relates the photospheric velocity to the Vabsolute magnitude, both evaluated at day $50\left(V_{\text {phot }}(50 \mathrm{~d}) \propto\right.$ $L_{\text {plateau }}^{0.33(0.04)}$, Hamuy 2003). Second, the tight relation found between the photospheric velocity at 50 days determined from the $\mathrm{Fe}$ II features and the velocity at a given time $t$. The best fit equation, based on a type IIP sample study, is: $V_{\text {phot }}(50 \mathrm{~d})=$ $V(t) *(t / 50)^{0.464 \pm 0.017}$ (Nugent et al. 2006).

For SN $2005 \mathrm{cs}$, we obtain estimates of $V_{\text {phot }}(50 \mathrm{~d})$ of $\sim 2600$ and $2315 \mathrm{~km} \mathrm{~s}^{-1}$ applying the two described methods. For SN 2005ay, since we do not have an early estimate of the Fe II velocities we rely only on the photospheric velocity recovered from the first method. We find $V_{\text {phot }}(50 \mathrm{~d}) \simeq 2980 \mathrm{~km} \mathrm{~s}^{-1}$ for the shorter distance and $\simeq 4070 \mathrm{~km} \mathrm{~s}^{-1}$ for the larger distance. The resulting parameters derived using both sets of analytical models are summarized in Table 7 for both SNe. These estimates, although inevitably suffering from various uncertainties, indicate that the two SNe, in particular SN 2005cs, represent examples where all three physical parameters, velocity, energy and ${ }^{56} \mathrm{Ni}$ mass are lower than average, a correlation not always observed in SNe IIP. Furthermore, recent results using pre-explosion imaging of SN 2005cs provide constraints on the progenitor main sequence mass (Maund et al. 2005). The authors found that the initial mass of the star is constrained to be $M_{\text {ZAMS }}=9_{-2}^{+3} M_{\odot}$, which is consistent within the uncertainties with the results of the present work.

We conclude that both type IIP SNe 2005ay with the shorter adopted distance, and $2005 \mathrm{cs}$ have lower than average luminosities, with SN 2005ay being slightly brighter and the shape of its light curves being more typical of SNe IIP. Adopting the larger distance possibility increases the luminosities and consequently the amount of the ejected ${ }^{56} \mathrm{Ni}$. SN $2005 \mathrm{cs}$ is distinguished by a slight brightening in the $V, R, I$ bands during the second half of the plateau stage, and by a low expansion velocity. Combining these results with the derived data on the progenitor stars, 
we suggest that these events resulted from the outburst of supergiants towards the lower end of the range of accepted progenitor masses with low explosion energies and low ${ }^{56} \mathrm{Ni}$ yields.

Acknowledgements. This research has made use of the Lyon-Meudon Extragalactic Database (LEDA) and of the Canadian Astronomy Data Centre. The authors are grateful to N. N. Pavlyuk and I. M. Volkov, who carried out some observations, to S. V. Antipin and K. Sokolovskij for help in the observations and to $\mathrm{O}$. S. Bartunov for help with computing facilities. The work of D. Yu. Tsvetkov was partly supported by RFBR grant 05-02-17480. A. Elmhamdi is grateful to David Branch for the use of the SYNOW code. I. J. Danziger was supported in this work by a MIUR-Cofin grant from the Italian Ministry of Education. We thank the referee, M. Della Valle, for the useful comments.

\section{References}

Baron, E., Branch, D., Hauschildt, P. H., et al. 2000, ApJ, 545, 444

Bouchet, P., \& Danziger, I. J. 1993, A\&A, 273, 451

Cardelli, J. A., Clayton, G. C., \& Mathis, J. S. 1989, ApJ, 345, 245

Catchpole, R. M., Whitelock, P. A., Feast, M. W., et al. 1988, MNRAS, 231, 75

Chevalier, C., \& Ilovaisky, S. A. 1991, A\&AS, 90, 225

Elmhamdi, A., Danziger, I. J., Chugai, N., et al. 2003a, MNRAS, 338, 939

Elmhamdi, A., Chugai, N., \& Danziger, I. J. 2003b, A\&A, 404, 1077
Elmhamdi, A., Danziger, I. J., Branch, D., et al. 2006, A\&A, 450, 305 Feldmeier, J. J., Ciardullo, R., \& Jacoby, G. H. 1997, ApJ, 479, 231 Hamuy, M. 2003, ApJ, 582, 905

Hamuy, M., Pinto, P. A., Maza, J., et al. 2001, ApJ, 558, 615

Kloehr, W. 2005, IAU Circ., 8553

Landolt, A. 1992, AJ, 104, 340

Leonard, D. C., Filippenko, A. V., Gates, E. L., et al. 2002a, PASP, 114, 35

Leonard, D. C., Filippenko, A. V., Li, W., et al. AJ, 2002b, 124, 2490

Li, W. 2005, IAU Circ., 8553

Li, W., Van Dyk, S. D., Filippenko, A. V., et al. 2006, ApJ, 641, 1060 Litvinova, I. Y., \& Nadyozhin, D. K. 1985, SVAL, 11, 145

Maund, J. R., Smartt, S. J., \& Danziger, I. J. 2005, MNRAS, 364, 33

Modjaz, M., Kirshner, R., Challis, P., \& Hutchins, R. 2005, IAU Circ., 8555

Nugent, P., Sullivan, M., Ellis, R., et al. 2006, ApJ, 645, 841

Popov, D. V. 1993, ApJ, 414, 712

Richardson, D., Branch, D., Casebeer, D., et al. 2002, AJ, 123, 745

Rich, D. 2005, IAU Circ., 8500

Richmond, M. W., Van Dyk, S. D., Ho, W., et al. 1996, AJ, 111, 327

Saha, A., Sandage, A., Tammann, G. A., et al. 2001, ApJ, 562, 314

Schlegel, D., Finkbeiner, D., \& Davis, M. 1998, ApJ, 500, 525

Schmidt, B. P., Kishner, R. P., \& Eastman, R. G. 1992, ApJ, 395, 366

Stetson, P. 2002, PASP, 112, 925

Taubenberger, S., Benetti, S., Harutyunyan, A., \& Zurita, A. 2005, IAU Circ., 8502

Yamaoka, H., \& Itagaki, K. 2005, IAU Circ., 8502 